\title{
SUBNORMAL SUBGROUPS AND FORMATION PROJECTORS
}

\author{
PETER FÖRSTER
}

(Received 25 January 1985)

Communicated by H. Lausch

\begin{abstract}
We discuss intersections of subnormal subgroups and formation projectors in finite (not necessarily soluble) groups.

1980 Mathematics subject classification (Amer. Math. Soc.): 20 D 10.

In [8] Huppert has shown that $F \cap N M=(F \cap N)(F \cap M)$ for each $\mathscr{m}$ projector $F$ and all normal subgroups $N, M$ of a finite soluble group, and he has derived some consequences of this property of Thprojectors. Apart from a few miscellaneous results (in Section 1) and a general lemma on Schunck class projectors (in Section 2), in the present paper we are mainly concerned with improving Huppert's results from Section 2 of [8] and generalising them to arbitrary finite groups, for which purpose we have to apply the theory of projectors in finite groups as developed in [3]. Therefore the reader is assumed to be familiar with definitions and results from [3]; furthermore, the notation used in the present paper is as explained in [3].
\end{abstract}

\section{Subnormal subgroups and Schunck class projectors}

One of the main results of [3] (namely, 4.2) states that $\operatorname{Proj}_{\mathscr{H}}^{+}(G) \neq \varnothing$ for any Schunck class $\mathscr{H}$ and each group $G$, where

$$
\begin{array}{r}
\operatorname{Proj}_{\mathscr{H}}^{+}(G)=\left\{H \leqslant G \mid N_{0}, N_{1}, \ldots, N_{m} \leqslant G, N_{0} \unlhd G, N_{i+1} \unlhd H N_{i}\right. \\
\left.(i=0, \ldots, m-1) \Rightarrow H \in \operatorname{Proj}_{\mathscr{H}}\left(H N_{m}\right)\right\} .
\end{array}
$$

() 1987 Australian Mathematical Society 0263-6115/87 \$A2.00+0.00 
Basically, the definition of $H \in \operatorname{Proj}_{\mathscr{W}}^{+}(G)$ says that firstly, $H \in \operatorname{Proj}_{\mathscr{H}}(H N)$ whenever $N \unlhd G$, and secondly, that this property is inherited by $H, H N$ (in place of $H, G)$. Here we shall prove the following refinement.

1.1. Proposition. Let $\mathscr{H}$ be a Schunck class, suppose that $G$ is a group with subnormal subgroup $S$, and let $H \in \operatorname{Proj}_{\mathscr{H}}^{+}(G)$. Then $H \in \operatorname{Proj}_{\mathscr{X}}^{+}(\langle H, S\rangle)$.

Proor. By way of contradiction assume that $G$ is a counterexample of least order, and let $M$ be a minimal normal subgroup of $G$. From $H M / M \in$ $\operatorname{Proj}_{\mathscr{P}}^{+}(G / M)$ and from $S M / M \unlhd \unlhd G / M$ we get

$$
H M / M \in \operatorname{Proj}_{\mathscr{E}}^{+}(\langle H, S\rangle M / M) .
$$

As $H \in \operatorname{Proj}_{\mathscr{H}}^{+}(H M)$, this together with [3,3.3] yields that $H \in \operatorname{Proj}_{\mathscr{H}}^{+}(\langle H, S\rangle M)$ for all minimal normal subgroups $M$ of $G$. In case that $\langle H, S\rangle M<G$ for some $M$, the desired contradiction is a consequence of our choice of $G$. Therefore, suppose that $\langle H, S\rangle M=G \neq\langle H, S\rangle$ for each minimal normal subgroup $M$ of $G$. By [3, 1.1], $G$ is necessarily primitive. In addition, if $G \in \mathscr{P}_{\mathrm{I}} \cup \mathscr{P}_{\mathrm{III}}$, then $\langle H, S\rangle$ is a maximal subgroup of $G$ which complements each minimal normal subgroup. In view of the canonical isomorphism $G / M=\langle H, S\rangle M / M \cong\langle H, S\rangle$, a contradiction emerges from (*). Hence $G \in \mathscr{P}_{\mathrm{II}}$. Then from $S \cap M \unlhd \unlhd M$ we see that either $S=1$, or that $S \cap M$ contains one of the simple direct factors of $M$, in which case $G=\langle H, S\rangle M=\langle H, S\rangle$. In both cases, however, $H$ clearly belongs to $\operatorname{Proj}_{\mathscr{K}}^{+}(\langle H, S\rangle)$.

Recall that a Fitting class is a class $\mathscr{F}$ of finite groups which is closed under taking subnormal subgroups and normal products; moreover, the set of $\mathscr{F}$-injectors of a finite group $G$ is defined to be

$$
\operatorname{Inj}_{F}(G)=\left\{F \leqslant G \mid F \cap S \in \operatorname{Max}_{\mathscr{F}}(S) \text { for each } S \unlhd \unlhd G\right\} \text {. }
$$

Clearly, if $\operatorname{Inj}_{\mathscr{F}}(G) \neq \varnothing$ for all $G \in \mathscr{E}$, then $\mathscr{F}$ must be a Fitting class, whereas the converse apparently does not hold true.

1.2. THEOREM. Let $\mathscr{F}$ be a Schunck class as-well as a Fitting class. Then $\operatorname{Proj}_{\mathscr{F}}(G)=\operatorname{Inj}_{\mathscr{F}}(G)$ for each $G \in \mathscr{E}$ if and only if $\mathscr{F}=\{1\}, \mathscr{F}=\mathscr{E}_{p}$ for some prime $p$, or $\mathscr{F}=\mathscr{E}$.

Proof. Clearly, $\mathscr{F}=Q \mathscr{F}=S_{n} \mathscr{F}=\mathscr{F}^{2}$ (where $\mathscr{F}=\mathscr{F}^{2}$ is a consequence of $\operatorname{Proj}_{\mathscr{F}}=\operatorname{Inj} \mathrm{j}_{\mathscr{F}}$ ); and any class $\mathscr{F}$ satisfying these conditions may be written as $\mathscr{F}=\mathscr{E}_{\mathscr{T}}$, the class of all finite groups all of whose composition factors belong to $\mathscr{X}$, where $\mathscr{X}$ is the class of all groups occurring as composition factors of groups in $\mathscr{F}$. We aim to show that either $\mathscr{X}=\varnothing, \mathscr{X}=\left\{C_{p}\right\}$ for some prime $p$, or $\mathscr{X}$ comprises all finite simple groups (including those of prime order). 
It is well known that for each non-abelian finite simple group $E$, and for every prime $p$ dividing $|E|$, there is a group $E_{\Phi, p}$ such that $E_{\Phi, p} / \Phi\left(E_{\Phi, p}\right) \cong E$, and $\Phi\left(E_{\Phi, p}\right) \neq 1$ is a $p$-group (see, for example, [6]). Therefore, if $\pi$ is the set of all primes dividing the orders of $\mathscr{F}_{\text {groups, }}$ then $E_{\Phi} \mathscr{F}=Q \mathscr{F}=S_{n} \mathscr{F}=N_{0} \mathscr{F}=\mathscr{F}$ shows that $\mathscr{E}_{p} \subseteq \mathscr{F}$ for all $p \in \pi$, and then $\mathscr{F}^{2}=\mathscr{F}$ gives $\mathscr{S}_{\pi} \subseteq \mathscr{S}_{\pi} \mathscr{F}=\mathscr{F} \subseteq \mathscr{E}_{\pi}$.

Next we claim that for any simple group $E, \operatorname{Max}_{\mathscr{F}}(E)$ is a set of isomorphic subgroups of $E$; and we observe that it suffices to prove this assertion in case that $|\pi| \geqslant 2$. As soon as this assertion will have been proved, the proof of the theorem can be completed as follows. From $\mathscr{S}_{\pi} \subseteq \mathscr{F} \subseteq \mathscr{E}_{\pi}$ and the assertion, one derives easily that $\operatorname{Max}_{F}(E)=\operatorname{Inj}_{\mathscr{F}}(E)$ coincides with $\operatorname{Hall}_{\pi}(E)$, whence $|\pi| \geqslant 2 \mathrm{im}$ plies that $\pi$ contains all primes (cf. [4, 4.1], which, by virtue of the proof given in [4], still holds under the present weaker hypothesis). This in turn gives $E \in$ $\operatorname{Hall}_{\pi}(E)=\operatorname{Inj}_{\mathscr{F}}(E) \subseteq \mathscr{F}$ for every finite simple group $E$, and we are done.

In order to prove the above claim we assume that $E$ is a simple (necessarily

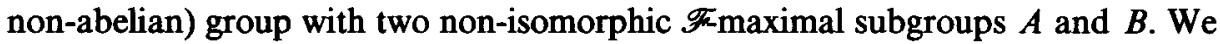
shall consider the regular wreath product

$$
X=E \sim C_{p}=C_{p} E^{*} \in \mathscr{P}_{\mathrm{II}}, \quad p \in \pi,
$$

and we shall employ the usual notations; specifically, if $D \leqslant E$, then $D_{i}$ denotes the subgroup of $E_{i}$ (the $i$ th component of the base group $E^{*}=E_{1} \times \cdots \times E_{p}$ ) which is isomorphically mapped onto $D$ by the canonical isomorphism from $E_{i}$ to $E$. Now note that $\left\{1, E^{*}, X\right\}$ is the set of all normal subgroups of $X$. Hence any $\mathscr{F}$ maximal subgroup of $X$ containing an $\mathscr{F}_{\text {injector }} E^{*}$ is an $\mathscr{F}_{\text {injector of }}$

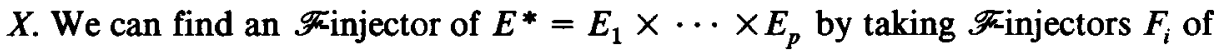
$E_{i}(i=1, \ldots, p)$ and forming their direct product: this assertion follows easily from $\mathscr{F}=Q \mathscr{F}$. Furthermore, the $\mathscr{T}$ injectors of the simple group $E$ are precisely

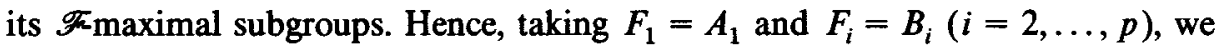
see that there is some $F \in \operatorname{Inj}_{\mathscr{F}}(X)$ such that $F \cap E^{*}=F_{1} \times \cdots \times F_{p}=A_{1} \times$ $B_{2} \times \cdots \times B_{p}$. From $X / E^{*} \cong C_{p} \in \mathscr{F}$ we get that $X=F E^{*}$, whence $F$ permutes transitively the simple direct factors of $E^{*}$, and so acts transitively on $\left\{F_{1}, \ldots, F_{p}\right\}$. This yields the contradiction that $B \cong B_{2}=A_{1}^{f} \cong A$ for some $f \in F$.

Observe that in the above theorem we did not require $\mathscr{F}$ projectors (or Frinjectors) to form a set of conjugate subgroups.

The next theorem generalises results of Huppert [8] and Ti Yen [13] on finite soluble groups.

1.3. THEOREM. For a Schunck class $\mathscr{H}$ the following three statements are equivalent.

(i) $\mathscr{H}=R_{0} \mathscr{H}$.

(ii) $H \cap N_{1} N_{2}=\left(H \cap N_{1}\right)\left(H \cap N_{2}\right)$ whenever $H \in \operatorname{Proj}_{\mathscr{H}}(G)$ and $N_{1}, N_{2} \unlhd G$. 
(iii) $H \cap N_{1} N_{2}=\left(H \cap N_{1}\right)\left(H \cap N_{2}\right)$ whenever $G$ is a group such that $\operatorname{Cov}_{\mathscr{H}}(G)$ $\neq \varnothing, H \in \operatorname{Cov}_{\mathscr{H}}(G)$, and $N_{1}, N_{2} \unlhd G$.

Proof, (i) $\Rightarrow$ (ii). This is shown in much the same way as $[8,2.1]$, except that one has to appeal to [3,6.1a] in order to show that $H \in \operatorname{Proj}_{\mathscr{H}}(G)$ and $N \unlhd G$ imply that $H \in \operatorname{Proj}_{\mathscr{H}}(H N)$. Indeed, in the crucial step of the proof this property of projectors for saturated formations is necessary in order to derive that $G=H N_{1}=H N_{2}$, after $N_{1} \cap N_{2}=1$ has been seen by means of a rather formal argument, using induction on $|G|$.

(ii) $\Rightarrow$ (iii). This is trivial.

(iii) $\Rightarrow$ (i). Assume that $R_{0} \mathscr{H} \neq \mathscr{H}$ and choose $G \in R_{0} \mathscr{H} \backslash \mathscr{H}$ of least order. Then there are two distinct minimal normal subgroups of $G, M_{1}$ and $M_{2}$ say, such that $G / M_{i} \in \mathscr{H}(i=1,2)$. Moreover, there exists $N \unlhd G$ such that $G / N \in$ $b(\mathscr{H})$. Clearly $M_{i} N / N$ is a minimal normal subgroup of $G / N$, and $M_{i} N=M_{i} \times$ $N$.

If $G / N \in \mathscr{P}_{\text {I }}$, then we let $H$ be a maximal subgroup of $G$ with core $(H)=N$, and from $G=H M_{i}$ we see that $H \in \operatorname{Proj}_{\mathscr{H}}(G)$ (using [3, 5.6a]). Thus $H \in$ $\operatorname{Cov}_{\mathscr{H}}(G)$ is a consequence of maximality of $H$ in $G$. This yields the contradiction that $H \cap M_{1} M_{2} \geqslant N \cap M_{1} M_{2} \neq 1=\left(N \cap M_{1}\right)\left(N \cap M_{2}\right)=\left(H \cap M_{1}\right)(H \cap$ $M_{2}$ ). A similar argument applies if $G / N \in \mathscr{P}_{\mathrm{III}}$, since then $H$ again complements both $M_{1}$ and $M_{2}$ and is maximal in $G$. Thus $G / N \in \mathscr{P}_{\mathrm{II}}$, and we get a contradiction as follows (cf. $[3,1.1 \mathrm{~b}])$ :

$$
N=C_{G}\left(M_{i} N / N\right) \geqslant M_{3-i} N=M_{i} N \quad(i=1,2) .
$$

In view of the above theorem, it is clear that for any saturated formation $\mathscr{F}$, each finite group $G$ has a unique largest normal subgroup $\mathbf{T}_{\mathscr{F}}(G)$ the intersection of which with each element of $\operatorname{Proj}_{\mathscr{F}}(G)$ is 1 ; note that $\operatorname{Proj}_{\mathscr{F}}^{+}=\operatorname{Proj}_{\mathscr{F}}$ for saturated formations $\mathscr{F}[3,6.1 \mathrm{a}]$. Clearly $\mathbf{T}_{\mathscr{F}}(G)$ is a characteristic subgroup of G.

1.4. Corollary. $\mathbf{T}_{\mathscr{F}}(G) \leqslant G^{\mathscr{F}}, \mathbf{T}_{\mathscr{F}}(G) N / N \leqslant \mathbf{T}_{\mathscr{F}}(G / N)$, and $\mathbf{T}_{\mathscr{F}}(G / N)=$ $\mathrm{T}_{\mathscr{F}}(G) / N$ for all $N \unlhd G$ contained in $\mathrm{T}_{\mathscr{F}}(G)$.

Proof. Since $F \in \operatorname{Proj}_{F}(G)$ covers $G / G^{\mathscr{F}}$, we have

$$
\begin{aligned}
\mathbf{T}_{\mathscr{F}}(G) & =\mathbf{T}_{\mathscr{F}}(G) \cap \mathbf{T}_{\mathscr{F}}(G) G^{\mathscr{F}} \cap F G^{\mathscr{F}}=\mathbf{T}_{\mathscr{F}}(G) \cap\left(\mathbf{T}_{\mathscr{F}}(G) G^{\mathscr{F}} \cap F\right) G^{\mathscr{F}} \\
& =\mathbf{T}_{\mathscr{F}}(G) \cap\left(\mathbf{T}_{\mathscr{F}}(G) \cap F\right)\left(G^{\mathscr{F}} \cap F\right) G^{\mathscr{F}}=\mathbf{T}_{\mathscr{F}}(G) \cap G^{\mathscr{F}} \leqslant G^{\mathscr{F}} .
\end{aligned}
$$

If $N \unlhd G$, then

$$
\begin{aligned}
\mathbf{T}_{\mathscr{F}}(G) N / N \cap F N / N & =\left(\mathbf{T}_{\mathscr{F}}(G) N \cap F\right) N / N \\
& =\left(\mathbf{T}_{\mathscr{F}}(G) \cap F\right)(N \cap F) N / N=N / N
\end{aligned}
$$


if, in addition, $N \leqslant \mathbf{T}_{\mathscr{F}}(G)$, then we put $T / N=\mathbf{T}_{\mathscr{F}}(G / N)$ and get

$$
T \cap F \leqslant N \cap F \leqslant \mathbf{T}_{\mathscr{F}}(G) \cap F=1,
$$

whence $T \leqslant \mathbf{T}_{\mathscr{F}}(G)$.

Huppert [8] has asked whether there is a neat description of $\mathbf{T}_{\mathscr{F}}(G)$. Indeed, one might wonder whether $\mathbf{T}_{\mathscr{F}}(G)$ is a "pregroup functor" (see [12]); i.e., does there exist a "subgroup functor" $f_{\mathscr{F}}$ such that $\mathbf{T}_{\mathscr{F}}(G)=\operatorname{core}_{G}(F)$ for any $F \in f_{\mathscr{F}}(G)$ ? (A subgroup functor $f$ associates to every group $G$ a set $f(G)$ of subgroups of $G$ such that $f(G / N)=f(G) N / N$ for all $N \unlhd G$.) However, even for finite soluble groups there is no such $f_{\mathscr{s}}$ :

1.5. ExAmple. Let $G=\operatorname{SL}(2,3)$, and let $\mathscr{N}$ be the class of all nilpotent groups. Then there does not exist a subgroup $F$ of $G$ such that $\mathrm{T}_{\mathscr{N}}(G)=\operatorname{core}_{G}(F)$ and $\mathbf{T}_{\mathcal{N}}(G / \mathbf{Z}(G))=\operatorname{core}_{G / \mathbf{Z}(G)}(F \mathbf{Z}(G) / \mathbf{Z}(G))$.

As observed in [8], from 1.3 one gets the following

1.6. CoRollary. Let $\mathscr{F}$ be a saturated formation. For each $F \in \operatorname{Proj}_{\mathscr{F}}(G)$, define a map $\phi_{F}^{G}$ from the lattice of normal subgroups of $G$ to the lattice of normal subgroups of $F$ by $\phi_{F}^{G}(N)=F \cap N(N \unlhd G)$. Then $\phi_{F}^{G}$ is a lattice homomorphism.

Huppert [8] then goes on to give a partial answer to the question of surjectivity of $\phi_{F}^{G}$, which was raised in view of some results of Wielandt [14]. In Section 3 we shall give a generalisation of Huppert's $[8,2.7]$ (or rather of what emerges from Huppert's proof of $[8,2.7])$. Prior to that we have to add a result on the basic properties of projectors to those contained in [3].

\section{A lemma on projectors}

The introduction in [3] of $\operatorname{Proj}_{\mathscr{*}}^{+}(G)$, and the characterisation of Schunck classes $\mathscr{H}$ by the property that $\operatorname{Proj}_{\mathscr{H}}^{+}(G) \neq \varnothing$ for all groups $G$, was aimed at providing a substitute for $\operatorname{Cov}_{\mathscr{H}}(G)$ (which may be empty), so that in proofs of statements relating to Schunck classes and their projectors induction would become a more powerful tool: not only can one pass to quotients of $G$, one can also pass to a sufficiently large set of subgroups of $G$ when dealing with elements of $\operatorname{Proj}_{\mathscr{X}}^{+}(G)$ rather than $\operatorname{Proj}_{\mathscr{H}}(G)$. Here we supplement the results from [3] by showing that '+-projectors' of the subgroups $X$ of $G$ involved in the definition of $\operatorname{Proj}_{\mathscr{*}}^{+}(G)$ (i.e., '+-projectors' of those $X \leqslant G$ which are required to satisfy 
$H \in \operatorname{Proj}_{\mathscr{X}}(X)$ in order that $H \leqslant G$ be in $\operatorname{Proj}_{\mathscr{P}}^{+}(G)$ ), and the subgroups $X$ themselves, enjoy some more useful properties.

2.1. Proposition. Let $\mathscr{H}$ be a Schunck class, and let $X \leqslant G$ be such that

(i) $H \leqslant X$ for some $H \in \operatorname{Proj}_{\mathscr{*}}^{+}(G)$, and

(ii) $X=H N_{m}$, where $N_{1}, \ldots, N_{m} \leqslant G$ satisfy $N_{1} \unlhd G, N_{i+1} \unlhd H N_{i}(i=1, \ldots$, $m-1)$.

(a) $(H \in) \operatorname{Proj}_{\mathscr{X}}^{+}(X) \subseteq \operatorname{Proj}_{\mathscr{H}}^{+}(G)$, and $\operatorname{Proj}_{\mathscr{H}}(X) \subseteq \operatorname{Proj}_{\mathscr{H}}(G)$.

(b) If $Y \leqslant X$ is such that

(i') $K \leqslant Y$ for some $K \in \operatorname{Proj}_{\mathscr{*}}^{+}(X)$, and

(ii') $Y=K M_{n}$, where $M_{1}, \ldots, M_{n} \leqslant X$ satisfy $M_{1} \unlhd X, \quad M_{i+1} \unlhd K M_{i} \quad(i=$ $1, \ldots, n-1)$,

then we have $Y=K L_{m+n}$, where $L_{1}=N_{1}, \ldots, L_{m}=N_{m}, L_{m+1}=M_{1}, \ldots, L_{m+n}$ $=M_{n}$ satisfy $L_{1} \unlhd G, L_{i+1} \unlhd K L_{i}(i=1, \ldots, m+n-1)$.

(Note that the (trivial) special case of (b) when $H=K$ asserts just the defining property of $\operatorname{Proj}_{\mathscr{*}}^{+}(G)$ as a subset of $\operatorname{Proj}_{\mathscr{H}}(G)$.)

Proof. (a) Induction on $m$ justifies the assumption that $m=1$ : note that $H \in \operatorname{Proj}_{\mathscr{H}}^{+}\left(H N_{i}\right)(i=1, \ldots, m)$. That is to say, $X=H N$ for some $N \unlhd G$; in particular, $X / N=H N / N \in \operatorname{Proj}_{\mathscr{H}}^{+}(G / N)$. If $L \in \operatorname{Proj}_{\mathscr{W}}^{+}(X)$, then [3, 3.3] may be applied to show that $L \in \operatorname{Proj}_{\mathscr{P}}^{+}(G)$.

Since Proj ${ }_{\mathscr{H}}^{+} \leqslant \operatorname{Proj}_{\mathscr{H}}$, the same argument works with $L \in \operatorname{Proj}_{\mathscr{H}}(X)$ instead of $\operatorname{Proj}_{\mathscr{K}}^{+}(X)$.

(b) $X / N_{m}=H N_{m} / N_{m} \in Q\{H\} \subseteq \mathscr{H}$ and $K \in \operatorname{Proj}_{\mathscr{H}}^{+}(X)$ yield that $X=K N_{m}$. From $H N_{m-1}=H N_{m} N_{m-1}=X N_{m-1}$, i.e., $X=H\left(X \cap N_{m-1}\right)$, we conclude similarly that $X=K\left(X \cap N_{m-1}\right)$, and $K N_{m-1}=X N_{m-1}=H N_{m-1}$. More generally, induction proves that $K N_{i}=X N_{i}=H N_{i}, i=1, \ldots, m$. It remains to observe that $M_{1} \unlhd X=H N_{m}=K N_{m}$.

\section{Formation projectors and normal subgroups}

In this and in the next section, $\mathscr{F}$ shall always denote a saturated formation, $\chi(\mathscr{F})$ shall be its characteristic, and $\mathscr{F}(p)$ its full and integrated $p$-local definition.

Moreover, we shall frequently, and without further reference, exploit the fact that $\operatorname{Proj}_{\mathscr{F}}=\operatorname{Proj}_{\mathscr{F}}^{+}$, which follows from $b(\mathscr{F}) \cap \mathscr{P}_{\mathrm{III}}=\varnothing$ (cf. $\left.[3,1.1 \mathrm{~b}]\right)$.

Before stating our first main result, (namely, 3.3 below) we shall record a consequence of the situation that is dealt with in that theorem. 
3.1 LemMa. If $G$ is a group with the property that $F \cap G^{\mathscr{F}}=1$ for each $F \in \operatorname{Proj}_{\mathscr{F}}(G)$, then $\operatorname{Proj}_{\mathscr{F}}(G)=\operatorname{Cov}_{\mathscr{F}}(G)$.

Proof. Let $F \in \operatorname{Proj}_{F}(G)$; we must show that $F \in \operatorname{Cov}_{\mathscr{F}}(G)$. We may, of course, assume that $G^{\mathscr{F}} \neq 1$, and choose $M \leqslant G^{\mathscr{F}}$ minimal normal in $G$. By means of induction on $|G|,[3,3.3]$ applies, showing that we may, in fact, assume that $M=G^{\mathscr{F}}$; note that $(F M)^{\mathscr{F}} \leqslant M$. Now let $F \leqslant U \leqslant G$; we have to verify that $U=F U^{\mathscr{F}}$. Since $U / U \cap G^{\mathscr{F}} \cong U G^{\mathscr{F}} / G^{\mathscr{F}}=F G^{\mathscr{F}} / G^{\mathscr{F}}=G / G^{\mathscr{F}} \in \mathscr{F}$, it follows that $U^{\mathscr{F}} \leqslant U \cap G^{\mathscr{F}}$. If $U^{\mathscr{F}}=U \cap G^{\mathscr{F}}$, then Dedekind's identity gives the desired result, while $U^{\mathscr{F}}<U \cap G^{\mathscr{F}}$ forces any $\mathscr{F}$ projector $V$ of $U$ to intersect $U \cap G^{\mathscr{F}}$ non-trivially; any $F_{0} \in \operatorname{Max}_{\mathscr{F}}(G)$ containing $V$, however, is already an $\mathscr{F}$ projector of $G\left([3,5.5]\right.$; we have observed above that $\left.b(\mathscr{F}) \cap \mathscr{P}_{\text {III }}=\varnothing\right)$, yet $F_{0} \cap G^{\mathscr{F}} \geqslant V \cap\left(U \cap G^{\mathscr{F}}\right) \neq 1$.

Our proof of 3.1 clearly relies on the hypothesis being a condition on all Thprojectors, although we do not know of a counterexample against the corresponding statement with respect to a fixed $\mathscr{F}$ projector.

3.2. REMARK. If $F \cap G^{\mathscr{J}}=1$ for each $G \in \mathscr{E}$ and each $F \in \operatorname{Proj}_{\mathscr{F}}(G)$, then $\mathscr{F}=\{1\}$ or $\mathscr{F}=\mathscr{E}$.

PRoof. Unless $\mathscr{F}=\{1\}$, there exists a prime $p$ such that $C_{p}$, the group of order $p$, belongs to $\mathscr{F}$. Considering $\mathscr{F} \neq\{1\}$ only, we shall show that $b(\mathscr{F}) \cap \mathscr{P}_{\text {II }}=\varnothing$. This will yield the desired conclusion, for in this case $G \in \mathscr{F}$ implies that $E \sim G \in \mathscr{F}$ for any non-abelian simple group $E$ (where $\sim$ denotes the regular wreath product), and thus $G \in \mathscr{F}(q)$ for each prime $q$, which together with $b(\mathscr{F}) \cap \mathscr{P}_{\mathrm{II}}=\varnothing$ forces $b(\mathscr{F})$ to be empty.

If $G \in b(\mathscr{F}) \cap \mathscr{P}_{\text {II }}$ and $F \in \operatorname{Proj}_{\mathscr{F}}(G)$, then there exists a faithful, irreducible $\mathrm{GF}(p)[G]$-module $V$ such that $V_{F}$ possesses a trivial irreducible submodule $T$. Therefore, letting $H$ be the semidirect product $G V$, we have that $H^{\mathscr{F}}=\mathrm{S}(G) V$ and $F T \cap H^{\mathscr{F}}=T \neq 1$, and yet $F T \cong F \times C_{p} \in \mathscr{F}$ is contained in an $\mathscr{k}$ projector of $H$, which is a contradiction.

3.3. THEOREM. Let $G$ be a finite group. Then the following four statements are equivalent.

(i) $F \cap G^{\mathscr{F}}=1$ for any $F \in \operatorname{Proj}_{\mathscr{F}}(G)$.

(ii) $F \cap U^{F}=1$ whenever $F \in \operatorname{Proj}_{F}(G)$ and $F \leqslant U \leqslant G$.

(iii) $\phi_{F}^{G}$ is surjective for any $F \in \operatorname{Proj}_{F}(G)$, and $G$ does not involve a section $X / Y$ such that

(1) there exist $N_{1}, \ldots, N_{m} \leqslant G$ and $F^{*} \in \operatorname{Proj}_{\mathscr{F}}(G)$ satisfying $N_{1} \unlhd G, N_{i+1} \unlhd F^{*} N_{i}$ $(i=1, \ldots, m-1)$ and $X=F^{*} N_{m}$, and 
(2) (2a) $\mathbf{S}(X / Y)=\mathbf{S}\left(F^{*} Y / Y\right)=\left(F^{*} Y / Y\right) \cap(X / Y)^{*}$ is a minimal normal $p$-subgroup of $F^{*} Y / Y$ for some prime $p$, and $(X / Y)^{*} / \mathrm{S}(X / Y)$ is a minimal normal $p^{\prime}$-subgroup of $(X / Y) / \mathrm{S}(X / Y)$ with Aut $_{F^{*}}\left((X / Y)^{\mathscr{F}} / \mathrm{S}(X / Y)\right) \notin$ $\mathscr{F}(q)$ for all $q \in \pi\left([X / Y]^{\mathscr{F}} / \mathbf{S}[X / Y]\right)$, or (2b) $\mathbf{S}(X / Y)=(X / Y)^{F}$ is a non-abelian minimal normal subgroup of $X / Y$, and $\mathrm{S}\left(F^{*} Y / Y\right)=F^{*} Y / Y \cap \mathrm{S}(X / Y)$ is the unique minimal normal subgroup of $F^{*} Y / Y$.

(iv) $\phi_{F}^{G}$ is surjective for any $F \in \operatorname{Proj}_{\mathscr{F}}(G)$, and $G$ does not involve a section $X / Y$ such that (2) holds for some $F^{*} \in \operatorname{Proj}_{\mathscr{F}}(G)$ contained in $X$.

Proof. (i) $\Rightarrow$ (ii). This is a trivial consequence of $U^{\mathscr{F}} \leqslant U \cap G^{\mathscr{H}}$.

(ii) $\Rightarrow$ (iv). Surjectivity of $\phi_{F}^{G}$ is evident from $G=F G^{\mathscr{F}}$ and $F \cap G^{\mathscr{F}}=1$. If $Y \unlhd X \leqslant G$ and if $F^{*} \in \operatorname{Proj}_{F}(G)$, then we may apply 1.3 to get that

$$
F^{*} Y / Y \cap(X / Y)^{\mathscr{F}}=F^{*} Y / Y \cap X^{\mathscr{F}} Y / Y=\left(F^{*} \cap X^{\mathscr{F}}\right)\left(F^{*} \cap Y\right) Y / Y=1 \text {. }
$$

Hence (2) is not valid for $X, Y, F^{*}$. (Note that 3.1 ensures that 1.3 is indeed applicable, whence $F^{*} \in \operatorname{Proj}_{F}(X)$.)

(iv) $\Rightarrow$ (iii). (iii) is a special case of (iv).

(iii) $\Rightarrow$ (i). From 2.1 one deduces easily that the condition that $G$ must not involve a section $X / Y$ satisfying (1) and (2) is inherited by any $H \leqslant G$ such that $H=F^{\sharp} L_{n}$ for some $F^{\sharp} \in \operatorname{Proj}_{F}(G), L_{1} \unlhd G, L_{i+1} \unlhd F^{\sharp} L_{i}(i=1, \ldots, n-1)$. Since $\phi_{F}^{H}$ is clearly surjective whenever $F \leqslant H \leqslant G$ and $F \in \operatorname{Proj}_{\mathscr{F}}(G)$, we may apply 2.1a to get that $\operatorname{Proj}_{\mathscr{F}}(H) \subseteq \operatorname{Proj}_{F}(G)$ for all $H \leqslant G$, subject to the aforementioned condition, thus proving the surjectivity of $\phi_{F}^{H}$ for all $F \in \operatorname{Proj}_{\mathscr{F}}(H)$ for these subgroups $H$ of $G$. Therefore (iii) is inherited by all such subgroups $H$ of $G$ and is readily seen to be inherited by quotients of $G$ as well.

Now choosing $G$ as a counterexample of least order, with $\mathscr{T}$ projector $F$ such that $F \cap G^{\$} \neq 1$, we see that

$F^{*} K / K \cap(H / K)^{\mathscr{F}}=K / K$ (or equivalently, $F^{*} \cap H^{\mathscr{F}} \leqslant$

$K$ ) whenever $H / K$ is a proper section of $G$ such that

(*) $\quad H=F^{\sharp} L_{n}$ for suitable $L_{1}, \ldots, L_{n} \leqslant G$, where $F^{\sharp} \in \operatorname{Proj}_{\mathscr{F}}(G)$ satisfies $L_{1} \unlhd G, \quad L_{i+1} \unlhd F^{\sharp} L_{i}(i=1, \ldots, n-1)$, and $F^{*} \in$ $\operatorname{Proj}_{\mathscr{F}}(H)$.

As in Huppert's proof of $[8,2.7]$, this together with 1.3 can be applied to infer that

$$
S=S(G) \text { is minimal normal in } G \text {, and } F \cap S=F \cap G^{\mathscr{F}} \neq 1 \text {; }
$$

(**) moreover, $F \cap \mathbf{S}(G)=\mathbf{S}(F)$ is the unique minimal normal subgroup of $F$.

Next we claim that 
Assume this to be false, and note that $S<G^{\mathscr{s}}$ : otherwise a contradiction against the Galois-Ore Theorem (in case that $S$ is abelian) or the non-existence of a section $X / Y$ satisfying (1) and (2b) (in case that $S$ is non-abelian) would emerge; in the latter case (**) has to be applied. Therefore we may consider a normal subgroup $N$ of $G$ such that $S<N<G^{\mathscr{F}}$. We put $H=F N$ and let $E$ be the characteristic of $S$ (i.e., the simple component of $S$ ). In view of $(*), F S<G$ and $F \in \operatorname{Proj}_{\mathscr{F}}(F S)$ yield that $F S=F(F S)^{\mathscr{F}}$ and $F \cap(F S)^{\mathscr{F}}=1$; in particular, $S=(F \cap S)(F S)^{\mathscr{F}}$ is a split extension. Hence $F \cap S \cong S /(F S)^{\mathscr{F}}$ is a direct product of copies of $E$. The same argument may be applied to $H$ instead of $F S$, giving $H=F H^{\mathscr{F}}, F \cap H^{\mathscr{F}}=1, N=(F \cap N) H^{\mathscr{F}}$ (where $\left.F \cap N=F \cap S\right),(F$ $\cap N) \cap H^{\mathscr{F}}=1$, and $N / H^{\mathscr{F}} \cong F \cap N=F \cap S \in D_{0}\{E\}$. Therefore $N^{D_{0}\{E\}}$ is a normal subgroup of $G$ contained in $H^{\mathscr{F}}$, whence $H^{\mathscr{F}} \cap S=(F S)^{\mathscr{F}}<S$ together with (**) forces $N^{D_{0}\{E\}}$ to be trivial; that is to say, $N \cong E \times \cdots \times E$.

Case 1. $E$ is a group of prime order $p$.

Now $\mathbf{S}(F)$, the unique minimal normal subgroup of $F$, is a $p$-group, whence $\mathbf{O}_{p^{\prime}}(F)=1$ and $F \in \mathscr{F}(p)$. However, we have that $H=F N$, where $F \in$ $\operatorname{Proj}_{\mathscr{F}}(H)$ and $N \leqslant \mathrm{O}_{p}(H)$, from which we see that $H=F N \in \mathscr{E}_{p} \mathscr{F}(p)=$ $\mathscr{F}(p) \subseteq \mathscr{F}$ coincides with $F$, which violates $F \cap N=F \cap G^{\mathscr{F}}=F \cap S \leqslant S<N$ $(\operatorname{see}(* *))$.

Case 2. $E$ is non-abelian.

Now $N \cong E \times \cdots \times E$ is necessarily a direct product of minimal normal subgroups of $G$, which is a contradiction against $\mathbf{S}(G)=S<N$.

Thus (***) holds, and we are left to show that $S$ is abelian, and that $G^{F} / S$ is a $\pi(S)^{\prime}$-group. In view of $(* *)$ and $(* * *)$, this would produce a contradiction against (***), since in this case $X / Y=G / 1$ would satisfy (1) and (2a), as we shall see below.

First assume that $S$ is non-abelian, say of characteristic $E$. We have shown above that $F \cap S \in Q\{S\} \subseteq D_{0}\{E\}$, and so we may infer from (**) that $F \in \mathscr{F}(q)$ for each $q \in \pi(E)$. Since we could have chosen $F$ subject to the condition that $\left|F \cap G^{\mathscr{F}}\right|$ be maximal we obtain a contradiction by considering some $F_{0} \in \operatorname{Proj}_{\mathscr{F}}\left(\mathrm{N}_{F S}(P)\right)$ for a non-trivial $P \in \operatorname{Syl}_{p}\left((F S)^{\mathscr{F}}\right)$. Indeed, $F_{0}$ covers $F S /(F S)^{\mathscr{F}} \cong F \in \mathscr{F}(p)$ (for it covers $\left.\mathbf{N}_{F S}(P) / \mathbf{N}_{F S}(P) \cap(F S)^{\mathscr{F}} \cong F S /(F S)^{\mathscr{F}}\right)$, it intersects $(F S)^{\mathscr{F}}$ non-trivially, and it is contained in an $\mathscr{m}$ projector of $F S$ and thus of $G$ (cf. [3, 5.5]).

This proves that $S$ is an elementary abelian $p$-group for some prime $p$. Consequently, $F S \in \mathscr{E}_{p} \mathscr{F}(p) \subseteq \mathscr{F}$, and hence $F S=F$.

Further, as $F \in \mathscr{F}(p)$, a similar argument applied to $G^{\mathscr{F}} / S$ in place of $S$ yields that the former group is a $p^{\prime}$-group: simply consider a minimal supplement 
$H / P$ of $\left(\mathbf{N}_{G}(P) \cap G^{F}\right) / P$ in $\mathbf{N}_{G}(P) / P$, where $P / S$ is a Sylow $p$-subgroup of $G^{\mathscr{F}} / S$, and then observe that $\left(H \cap G^{\mathscr{F}}\right) / P \leqslant \Phi(H / P)$ and $H / H \cap G^{\mathscr{F}} \cong F / S$.

Finally, another argument of the same kind shows that $\operatorname{Aut}_{F}\left(G^{\mathscr{F}} / S\right) \notin \mathscr{F}(q)$ for each $q \in \pi\left(G^{\mathscr{F}} / S\right)$.

3.4. REMARK. In statement (iv) of 3.3, condition (2a) may be modified by writing ' $q$-subgroup $(q \neq p$ a prime $)$ ' instead of ' $p$ '-subgroup'. This can be shown by means of 3.1 together with the elementary properties of projectors and covering subgroups for saturated formations described in [3, Sections 3 and 5].

3.5. COROLLARY. Suppose that $\mathscr{F}$ admits a local definition $f$ such that $f(p)=f(q)$ for all primes $p, q$.

(a) $F \cap G^{\mathscr{F}}=1$ for each $F \in \operatorname{Proj}_{\mathscr{F}}(G)$ if and only if for each $F \in \operatorname{Proj}_{\mathscr{F}}(G)$, $\phi_{F}^{G}$ is surjective, and for every $X \leqslant G$ containing $F$ and satisfying $X=F N_{m}$ for suitable subgroups $N_{1}, \ldots, N_{m} \leqslant G$ such that $N_{1} \unlhd G, N_{i+1} \unlhd F N_{i}(i=1, \ldots, n-1)$, and for every $Y \unlhd X, X / Y$ is not a group in $b(\mathscr{F}) \cap \mathscr{P}_{\mathrm{II}}$ with $F Y / Y \cap S(X / Y)=$ $\mathrm{S}(F Y / Y)$ minimal normal in $F Y / Y$.

(b) If $G \in \mathscr{S}$ or $\mathscr{F} \subseteq \mathscr{S}$, then $F \cap G^{\mathscr{F}}=1$ for each $F \in \operatorname{Proj}_{\mathscr{F}}(G)$ if and only if $\phi_{F}^{G}$ is surjective for each $F \in \operatorname{Proj}_{\mathscr{F}}(G)$.

Proof. (a) is easily derived from 3.3, for a section $X / Y$ of $G$ satisfying (1) and (2a) would violate $f(p)=f(q)$ : the Frattini argument, applied to $H / Y \in$ Hall $_{p^{\prime}}\left((X / Y)^{\mathscr{F}} / \mathrm{S}(X / Y)\right)$ would yield that $X / Y, \quad F^{*} Y / Y \in \mathscr{P}_{\mathrm{I}}$, whence $\left(F^{*} Y / Y\right) / \mathbf{S}\left(F^{*} Y / Y\right) \in f(p)=f(q)$.

(b) If $G \in \mathscr{S}$, then $Q S\{G\} \cap \mathscr{P}_{\text {II }}=\varnothing$, whence the result is immediate from (a).

Now suppose that $\mathscr{F} \subseteq \mathscr{S}$. It will suffice to show that a group $H \in b(\mathscr{F}) \cap \mathscr{P}_{\text {II }}$ such that for any $F \in \operatorname{Proj}_{F}(H), F \cap \mathrm{S}(H)$ is the unique minimal normal subgroup of $F$ or is 1 , and $F \cap \mathrm{S}(H) \neq 1$ for at least one such $F$, cannot exist. By the way of contradiction, let $H$ be such a group and choose $F \in \operatorname{Proj}_{\mathscr{F}}(H)$ subject to the condition that $|F \cap \mathbf{S}(H)| \neq 1$ be minimal. Since $F \in \mathscr{F} \subseteq \mathscr{S}$, it follows that $\mathbf{S}(F)=F \cap \mathbf{S}(H)$ is an elementary abelian $p$-group for some prime p.

Put $K=\mathbf{N}_{H}(\mathbf{S}(F)) \geqslant F$. Then $F<K$ : indeed, otherwise $\mathbf{S}(F)=F \cap \mathbf{S}(H)=$ $K \cap \mathbf{S}(H)=\mathbf{N}_{\mathbf{S}(H)}(\mathbf{S}(F))$ would be a self-normalising abelian Sylow p-subgroup of $\mathbf{S}(H)=\mathbf{S}(H)^{\prime}$, which contradicts a well-known result of Burnside. Now we can choose $L \leqslant K$ containing $F$ as a maximal subgroup. Put $T=L \cap \mathrm{S}(H)$, and note that $T / \mathbf{S}(F)$ is a (necessarily minimal) normal subgroup of $L / \mathbf{S}(F)$ which is complemented by the maximal subgroup $F / \mathrm{S}(F)$. By Lemma 3.12 below, $F / \mathbf{S}(F) \in \mathscr{F} \subseteq \mathscr{S}$ implies that $T / \mathbf{S}(F)$ is elementary abelian, say of characteristic $q$. As $\mathbf{O}_{p^{\prime}}(F)=1, F \in \operatorname{Proj}_{F}(H)$ belongs to $\mathscr{F}(p)$, from which $q \neq p$ is 
immediate. Let $Q \in \operatorname{Syl}_{q}(T)$. By the Frattini argument, $L=\mathbf{N}_{L}(Q) T=$ $\mathbf{N}_{L}(Q) Q \mathbf{S}(F)=\mathbf{N}_{L}(Q) \mathbf{S}(F)$.

Case 1. $\mathbf{N}_{L}(Q)<L$.

In this case $F=\mathbf{N}_{F}(Q) \mathbf{S}(F) \neq \mathbf{N}_{F}(Q)$, and so the unique minimal normal subgroup $\mathbf{S}(F)$ of $F$ is complemented. Therefore $F$ is a primitive soluble group and, in particular, $F / \mathbf{S}(F)=F / \mathbf{C}_{F}(\mathbf{S}(F)) \in f(p)=f(q)$. We conclude that $L / \mathbf{S}(F)=(F / \mathbf{S}(F))(Q \mathbf{S}(F) / \mathbf{S}(F))$ is in $\mathscr{F}$, too. Hence any $F^{*} \in \operatorname{Proj}_{F}(L)$ covers $T / \mathrm{S}(F)$; without loss of generality $Q \leqslant F^{*}$ for some $F^{*} \in \operatorname{Proj}_{\mathscr{F}}(L)$. Clearly, $F^{*}$ is contained in an $\mathscr{g}$ projector $F^{\sharp}$ of $H[3,4.1]$. Moreover, $F^{\sharp} \cap$ $\mathbf{S}(H) \geqslant F^{*} \cap T \geqslant Q \neq 1$, whence $F^{\sharp} \cap \mathbf{S}(H)$ is a minimal normal $q$-subgroup of $F^{\sharp}$. Since $F^{\sharp}=F^{*}\left(\mathbf{S}(H) \cap F^{\sharp}\right)$, and since $F^{*}$ acts irreducibly on $Q \leqslant F^{\sharp} \cap$ $\mathbf{S}(H)$, we must have that $F^{\#} \cap \mathbf{S}(H)$ coincides with $Q$, i.e., $F^{*}=F^{\#} \in$ $\operatorname{Proj}_{\mathscr{F}}(H)$. Since $L / C$, where $C=\operatorname{core}_{L}\left(\mathbf{N}_{L}(Q)\right)$, is a primitive soluble group with minimal normal subgroup $\mathrm{S}(F) C / C$, and since $Q \cong Q C / C$ (for $Q \leqslant C$ would yield that $[Q, \mathbf{S}(F)]=1$ and $L=\mathbf{N}_{L}(Q) \mathbf{S}(F)=\mathbf{N}_{L}(Q)$ ), we get from Gaschütz [7] that $|Q|<|\mathbf{S}(F)|$. This yields a contradiction against our choice of $F: F^{*} \in \operatorname{Proj}_{\mathscr{F}}(H)$ satisfies $1 \neq\left|F^{*} \cap \mathbf{S}(H)\right|=|Q|<|F \cap \mathbf{S}(H)|$.

Case 2. $Q \unlhd L$.

In this case $L=F Q$ is a split extension. If $\mathbf{S}(F) \nless \Phi(F)$, then $F$ would be primitive, and an argument as before would show that $Q$ was an $\mathscr{\pi}$ central minimal normal subgroup of $L$, from which $L \in \mathscr{F}$ would follow. This contradicts $L>F \in \operatorname{Proj}_{\mathscr{F}}(H)$. Hence $\mathbf{S}(F) \leqslant \Phi(F)$. Since $\mathbf{S}(F) \unlhd K$, we have $\mathbf{S}(F) \leqslant \Phi(K)$, too. Now, if $\mathbf{S}(F) \notin \operatorname{Syl}_{p}(\mathbf{S}(H))$, we could have arranged for $L$ to be a subgroup of $\mathbf{N}_{H}\left(\mathbf{O}_{p}(F)\right.$ ) (which is contained in $\mathbf{N}_{H}(\mathbf{S}(F)$ ), for $\mathbf{S}(F)=$ $\left.\mathbf{O}_{p}(F) \cap \mathbf{S}(H)\right)$. Therefore we may assume that $\mathbf{S}(F) \in \operatorname{Syl}_{p}(\mathbf{S}(H))$, or that $L \leqslant \mathbf{N}_{H}\left(\mathbf{O}_{p}(F)\right)$. In the former case, $\mathbf{N}_{\mathbf{S}(H)}(\mathbf{S}(F))=\mathbf{S}(F) \times \mathbf{O}_{p^{\prime}}\left(\mathbf{N}_{\mathbf{S}(H)}(\mathbf{S}(F))\right)$, because $\mathbf{S}(F) \leqslant \Phi\left(\mathbf{N}_{H}(\mathbf{S}(F))\right)$ permits application of the Frattini argument, giving that a Hall $p^{\prime}$-subgroup of the ( $p$-soluble) group $\mathbf{N}_{\mathbf{S}(H)}(\mathbf{S}(F)$ ) is normal in $K$. Now we see again that $\mathbf{S}(F) \in \operatorname{Syl}_{p}(\mathbf{S}(H))$ is contained in $\mathbf{Z}\left(\mathbf{N}_{\mathbf{S}(H)}(\mathbf{S}(F))\right)$, which contradicts Burnside's Theorem as above. Thus we are left with the case $L \leqslant \mathbf{N}_{H}\left(\mathbf{O}_{p}(F)\right)$, in which we see that $L / \mathbf{C}_{L}(Q) \cong F / \mathbf{C}_{F}(Q) \in Q\left\{F / \mathbf{O}_{p}(F)\right\}$ $\subseteq Q R_{0} f(p)=f(p)=f(q)$. Consequently, $Q$ is $\mathscr{m}$ central in $L$, and we have $L \in \mathscr{F}$, which we have already seen to be impossible.

3.6. REMARK. A saturated formation $\mathscr{F}$ admits a local definition $f$ with $f(p)=$ $f(q)$ for all primes $p, q$, if and only if $\mathscr{F}=\mathscr{N} \mathscr{H}$ for a suitable formation $\mathscr{H}$. 
Proof. If $f(p)=f(q)$ for all primes $p, q$, then clearly $\mathscr{N} \mathscr{H} \subseteq L F(f)=\mathscr{F}$, where $\mathscr{H}=f(p) \cap \mathscr{F}$ for all primes $p$. Also $\mathscr{F} \cap b(\mathscr{N} \mathscr{H})=\varnothing$, since $G \in \mathscr{F} \cap$ $b(\mathscr{N} \mathscr{H}) \subseteq \mathscr{P}_{1} \cup \mathscr{P}_{\text {II }}\left(\mathscr{N} \mathscr{H}\right.$ is a saturated formation) should satisfy $G / \mathbf{C}_{G}(\mathbf{S}(G))$ $\in \mathscr{H}$. Conversely, if $\mathscr{F}=\mathscr{N} \mathscr{H}$, then we may take $f(p)=\mathscr{H}$ for all primes $p$.

Since $\operatorname{Proj}_{\mathscr{F}}(G)=\operatorname{Proj}_{\mathscr{F} \cap \mathscr{S}}(G)$ for all $G \in \mathscr{S}$, the part of $3.5 \mathrm{~b}$ dealing with $G \in \mathscr{S}$ is a (fairly easy) special case of the part dealing with $\mathscr{F} \subseteq \mathscr{S}$; in view of the conjugacy of all $\mathscr{k}$ projectors of a finite soluble group, the former result is just part (A) of Huppert $[8,2.7]$.

Part (B) of $[8,2.7]$ gives the same conclusion, but the condition on local definitions of $\mathscr{F}$ is replaced by the requirement that $\mathscr{F}$ be closed under taking subgroups and, in addition, contain $\operatorname{Aut}(H / K)$ whenever $H / K$ is a (soluble) chief factor of some $\mathscr{R}$ group. This hypothesis, however, requires either that $\mathscr{F}$ contain no non-supersoluble groups, or that $\mathscr{F}=\mathscr{E}$. Indeed, if a non-cyclic abelian group is a chief factor of an $\mathscr{m}$ group, then $\operatorname{GL}(n, p) \in \mathscr{F}$ for some prime $p$ and some natural number $n \neq 1$, and for each natural number $m$ we infer that $G_{m}=\mathrm{GL}(n, p) \times \dddot{m}_{m} \times \mathrm{GL}(n, p) \in \mathscr{F}(p)$. Consequently, $G_{m} V_{m} \in \mathscr{F}$ for every irreducible $\operatorname{GF}(p)\left[G_{m}\right]$-module $V_{m}$, and here $m$ can be chosen with $d=$ $\operatorname{dim}_{\mathrm{GF}(p)} V_{m}$ being arbitrarily large. Finally, $S_{d} \leqslant \mathrm{GL}(d, p) \in \mathscr{F}$ for any (sufficiently large) $d$ and $\mathscr{F}=S \mathscr{F}$ yield the desired conclusion: observe that either $\mathscr{F} \subseteq \mathscr{S}$ or that there is an $\mathscr{F}$ group with abelian chief factor of rank greater than 1 (e.g., a suitable Frattini extension of a group from $\mathscr{F} \cap \mathscr{P}_{\text {II }}$ ).

When $\mathscr{F}=\mathscr{E}$, Huppert's result is trivial, while the case when $\mathscr{F}$ consists of supersoluble groups can be handled easily, using 3.3.

We record another consequence of 3.3 ; a different proof of this result will be given in [2].

3.7. COROLlaRY. $\phi_{F}^{G}$ is surjective for each finite group $G$ and every $F \in \operatorname{Proj}_{F}(G)$ if and only if $\mathscr{F}=\{1\}$ or $\mathscr{F}=\mathscr{E}$.

Proof. Aiming at an application of 3.3 and 3.2, we shall prove that a triple $G$, $H=X / Y$, and $F=F^{*} X / Y$ as in condition (iii) of 3.3 cannot exist. If $H$ and $F$ are such groups, however, we may form the regular wreath product $H^{*}=E \sim H$, where $E=\operatorname{PSL}\left(2, p^{2}\right)$ for some $p \in \pi(\mathbf{S}(F))$. Let $P \in \operatorname{Syl}_{p}(E)$. Then $F P^{*} \in$ $\mathscr{E}_{P} \mathscr{F}(p) \subseteq \mathscr{F}$ is contained in an $\mathscr{F}$ projector $F^{\#}$ of $H^{*}$. Since $P$ is not contained in any non-soluble proper subgroup of $E$, from the surjectivity of $\phi_{F^{*}}^{H^{*}}$ it is easy to deduce by means of $[4,4.1]$ that $F^{\#}=F P^{*}$ with minimal normal subgroup $P^{*}$; but this is absurd, for we have $P^{*} \cong \oplus_{n} \mathrm{GF}(p)[F]$ with $n \neq 1$. 
An examination of the first part of the proof of 3.3 provides a proof of the following reduction theorem (from which the reader may derive an analogue of 3.5).

3.8. Corollary. Let $F \in \operatorname{Proj}_{F_{F}}(G)$. Then the following two statements are equivalent.

(i) $F \cap G^{\mathscr{F}}=1$.

(ii) $\phi_{F}^{G}$ is surjective, and $G$ involves no section $X / Y$ such that $F \in \operatorname{Proj}_{F}(X)$, and such that

either $\mathbf{S}(X / Y)$ is minimal normal in $X / Y, \mathbf{S}(F Y / Y)$ is minimal normal in $F Y / Y$, and $(X / Y)^{g} / \mathrm{S}(X / Y)$ is minimal normal in $(X / Y) / \mathrm{S}(X / Y)$, or $X / Y \in b(\mathscr{F}) \cap \mathscr{P}_{\mathrm{II}}$ and $\mathrm{S}(F Y / Y)=F Y / Y \cap \mathrm{S}(X / Y)$ is minimal normal in $F Y / Y$.

3.9. Examples. (1) Let $\mathscr{F}=h(B)(=\{F \in \mathscr{E} \mid B \notin Q\{F\}\})$, where $B \in \mathscr{P}_{\text {II }}$ has a maximal subgroup $F$ complementing the non-abelian unique minimal normal subgroup $\mathbf{S}(B)$ of $B$. (Examples of such groups $B$ have been constructed in [5].) Then $F \in \operatorname{Proj}_{F}(B)$, and $F \cap B^{\mathscr{F}}=F \cap S(B)=1$, but there is an $F^{*} \in \operatorname{Proj}_{F}(B)$ such that $F^{*} \cap B^{\mathscr{F}} \neq 1$.

By Kovács's Theorem in [5, Addendum], we have $B \cong E \sim_{N} F$, the twisted wreath product, where $F$ is a group with subgroups $X$ and $Y$ such that $Y \unlhd X$, $X / Y=E$ is simple, core $F_{F}(X)=1$, and $X \leqslant K$ whenever $K>Y$ is a subgroup of $H$ normalised by $N=\mathbf{N}_{F}(X) \cap \mathbf{N}_{F}(Y)$. (There are two misprints in the statement of Kovács's Theorem in [5]: in the first line, ' $X \unlhd Y$ ' should read ' $Y \unlhd X$ ', and in the third line, ' $X$ ' should read ' $N=\mathbf{N}_{H}(X) \cap \mathbf{N}_{H}(Y)$ '.)

As Lafuente [10] has observed, $F$ is necessarily a primitive group of type II, and $S(F)$ involves a section isomorphic to $E$ (consider $K=Y M$, where $M$ is a minimal normal subgroup of $F$ ). Now consider $\mathbf{N}_{B}(P)$, where $1 \neq P \in$ $\operatorname{Syl}_{p}(\mathbf{S}(B))$, and use the fact that $\mathbf{N}_{B}(P) / \mathbf{N}_{\mathbf{S}(B)}(P) \cong B / \mathbf{S}(B) \cong F \in \mathscr{F}(p)$.

(2) Let $\mathscr{F}$ be the class of all $p$-groups for a prime $p$, and let $E$ be a non-abelian finite simple group with Sylow $p$-subgroup of order $p$. Then $\phi_{P}^{E}$ is surjective for each $P \in \operatorname{Proj}_{\mathscr{F}}(E)=\operatorname{Syl}_{p}(E)$, but $E^{\mathscr{F}}=E$, and $P \cap E^{\mathscr{F}} \neq 1$.

Therefore the hypothesis concerning the sections $X / Y$ of $G$ which satisfy (1) and $(2 \mathrm{~b})$ in our Theorem 3.3 is not redundant. Similar examples may be found to show that the hypothesis concerning sections which satisfy (1) and (2a) must not be omitted.

Incidentally, 3.9(2) also provides a counterexample to 2.8 of Huppert [8]. Adding the hypothesis of $p$-solubility, however, leads to a correct statement:

3.10. Proposition. Let $F \in \operatorname{Proj}_{\delta_{p}}(G)=\operatorname{Syl}_{p}(G)$, where $G$ denotes a p-soluble group. If $\phi_{F}^{G}$ is an epimorphism, then $G \in \mathscr{E}_{p^{\prime}} \mathscr{E}_{p} \mathscr{E}_{p^{\prime}}$. 
Proof. Since the hypothesis is inherited by quotients of $G$ (which we have seen previously), a counterexample $G$ of least order is a $p$-soluble group in $b\left(\mathscr{E}_{p^{\prime}}, \mathscr{E}_{p} \mathscr{E}_{p^{\prime}}\right)$, and it satisfies $O_{p^{\prime}}(G)=1$. Hence $G=H V$ is a split extension, where $H \in$ $\mathscr{E}_{p^{\prime}} \mathscr{E}_{p} \mathscr{E}_{p^{\prime}} \backslash \mathscr{E}_{p} \mathscr{E}_{p^{\prime}}$ acts faithfully and irreducibly on its module $V$ over $\mathrm{GF}(p)$. In order that $\phi_{F}^{G}$ be surjective, $V=\mathbf{O}_{p}(G) \leqslant F$ has to be cyclic, in which case $H \cong$ Aut $_{G}(V)$ is isomorphic to a $p^{\prime}$-group (namely, a subgroup of the cyclic group of order $p-1$ ). Thus we have obtained a contradiction.

To conclude this section, we briefly comment on the injectivity of $\phi_{F}^{G}$.

3.11. Proposition. The following statements are equivalent.

(i) $b(\mathscr{F})$ does not contain a primitive group the socle of which is complemented by a maximal subgroup.

(ii) $F \cap S(G) \neq 1$ for each $G \in b(\mathscr{F})$ and every $F \in \operatorname{Proj}_{\mathscr{F}}(G)$.

(iii) $\phi_{F}^{G}$ is injective for each $G \in \mathscr{F}$ and every $F \in \operatorname{Proj}_{\mathscr{F}}(G)$.

(Note that any of the above three conditions immediately implies that $b(\mathscr{F}) \subseteq$ $\mathscr{P}_{\mathrm{II} \cdot}$.)

ProOF. (i) $\Rightarrow$ (ii). Assume that $G \in b(\mathscr{F})$ is a group of least order such that $F \cap \mathbf{S}(G)=1$ for some $F \in \operatorname{Proj}_{\mathscr{F}}(G)$. Let $F<H \leqslant G$ with $F$ maximal in $H$. From $G=F \mathbf{S}(G)$ and $F \cap \mathbf{S}(G)=1$ we deduce that $H=F(H \cap \mathbf{S}(G))$, where $H \cap \mathrm{S}(G)$ is minimal normal in $H$ and intersects $F$ trivially. In this situation it is easy to see that $F \in \operatorname{Proj}_{\mathscr{F}}(H)$. Consequently, $H / \operatorname{core}_{H}(F) \in b(\mathscr{F})$ has a maximal subgroup $F / \operatorname{core}_{H}(F) \in \operatorname{Proj}_{\mathscr{F}}\left(H / \operatorname{core}_{H}(F)\right)$. This contradicts either (i) (if $H=G$ ) or the choice of $G$ as a minimal counterexample (if $H \neq G$ ).

(ii) $\Rightarrow$ (iii). Let the pair $F, G$ be a counterexample such that $|G|$ is minimal. Then there are $X, Y \unlhd G$ satisfying $F \cap X=F \cap Y$ and $X \neq Y$. Replacing $Y$ by $X \cap Y$, we see that $Y<X$, and then we may suppose that $Y=1$. Furthermore, as $F \cap X=F \cap Y=1$ and $F \in \operatorname{Proj}_{\mathscr{F}}(F X), X$ is minimal normal in $G=F X$, and $X=G^{F}$. Now it is easily seen that $G /$ core $_{G}(F)$ is primitive (although $F$ is not necessarily maximal in $G)$, and that it belongs to $b(\mathscr{F})$. Finally, upon observing that $G / \operatorname{core}_{G}(F)$ is a counterexample, too, we obtain a contradiction to (ii).

(iii) $\Rightarrow$ (i). This is trivial.

The following lemma contains some elementary information on those groups mentioned in 3.11(i) whose socle is non-abelian. (A more complete result, due to Kovács, is formulated in [5]; see also [9].)

3.12. LemMA. Let $G=F M$ be a group with maximal subgroup $F$ complementing the nonabelian minimal normal subgroup $M$ of $G$. Let $M=E_{1} \times \cdots \times E_{n}$ be the 
decomposition of $M$ as a direct product of simple groups $E_{i} \cong E$, and put $N=$ $\mathrm{N}_{F}\left(E_{1}\right)$. Then, to within isomorphism, we have $G=E \sim_{N} F$, the twisted wreath product with respect to the given action of $N$ on $E=E_{1}$. Moreover, $\operatorname{Aut}_{N}(E) \cong$ $N / \mathrm{C}_{N}(E)$ is isomorphic to a subgroup of $\operatorname{Aut}(E)$ which contains $\operatorname{Inn}(E)$ (and hence is non-soluble).

Proof. That $G \cong E \sim_{N} F$ is well known (cf. [11]). For the sake of simplicity, we assume that $G=E \sim_{N} F$, where $F$ is the canonical complement of the base group $E^{*}=M$ of $G$.

Put $H=N E \leqslant G$ and $\bar{H}=H / C$, where $C=\mathrm{C}_{N}(E)$. We shall adopt the usual bar convention with respect to subgroups of $H$. Now note that $E$ is minimal normal in $H$, for otherwise we could find a proper subgroup $D \sim_{N} F>F$ of $G\left(1 \neq D<E, N \leqslant \mathbf{N}_{H}(D)\right)$. The same argument establishes the maximality of $N$ in $H$ : simply observe that $X=N(E \cap X)$ and $N \leqslant \mathbf{N}_{H}(E \cap X)$ whenever $N \leqslant X \leqslant H$. Hence $\bar{H}$ is a primitive group ( $\bar{N}$ being a core-free maximal subgroup) with non-abelian simple minimal normal subgroup $\bar{E}$. By $[1,6.3]$, the maximality of $\bar{N}$ in $\bar{H}$ excludes the case that $\bar{E}$ is the unique minimal normal subgroup of $\bar{H}$. Therefore, in view of $\mathrm{C}_{N}(E)=\operatorname{core}_{H}(N)$, the result follows from $[3,1.1]$.

Combining 3.12 with 3.11 and the fact that $b(\mathscr{S}) \subseteq \mathscr{P}_{\mathrm{II}}$, we get

3.13. ExAmple. $\mathscr{F}=\mathscr{S}$, the saturated formation of all finite soluble groups, satisfies conditions (i)-(iii) of 3.11 .

\section{Formation projectors and subnormal subgroups}

In this last section we shall deal with the question of the extent to which the property of formation projectors discussed in 1.3 generalises to subnormal subgroups.

4.1. Proposition. If $F \cap\langle S, T\rangle=\langle F \cap S, F \cap T\rangle$ whenever $G \in \mathscr{E}, F \in$ $\operatorname{Proj}_{\mathscr{F}}(G)$ and $S, T \unlhd \unlhd G$, then $\mathscr{F}=\mathscr{F}(p)$ for any $p \in \chi(\mathscr{F})$.

Proof. Assume the result is false. Then there exist $p \in \chi(\mathscr{F})$ and $G \in$ $\mathscr{F} \backslash \mathscr{F}(p)$. Put $H=G^{\mathscr{F}(p)}$. Choose a non-abelian simple group $E$ with $p \| E \mid$. The regular wreath product $X=E \sim G \in \mathscr{P}_{\text {II }}$ can be written as a split extension $X=G E^{*}$, where $G \leqslant X$, and where $E^{*}=E_{1} \times \cdots \times E_{n}$, the base group, with $E_{i} \cong E$ simple, is minimal normal in $X$. Clearly, $E^{*}$ contains a $G$-invariant 
subdirect subgroup $E^{\sharp}=E(1) \times \cdots \times E(m)$ such that $E(j) \cong E(j=1, \ldots, m$ $=\mid G / H D$ and $G E^{\sharp}=E \sim_{G / H} G$ (to within isomorphism), where $\sim_{G / H}$ denotes the wreath product with respect to the permutation representation of $G$ on the cosets of $H$ in $G$. Taking $P(i) \in \operatorname{Syl}_{p}(E(i))$ with $G$-invariant $P(1) \times \cdots \times P(m)$, we find that

$$
F_{0}=G(P(1) \times \cdots \times P(m)) \in R_{0}\left\{\mathscr{E}_{p} \mathscr{F}(p) \cup \mathscr{F}\right\} \subseteq \mathscr{F} .
$$

Now choose an $\mathscr{F}$ maximal subgroup $F \geqslant F_{0}$ of $X$. Since $E^{*}=\mathbf{S}(X)$ is minimal normal in $X$, we have $F \in \operatorname{Proj}_{\mathscr{F}}(X)$, whence the hypothesis about $\mathscr{F}$ yields that

$$
F=G E^{*} \cap F=G\left(E^{*} \cap F\right)=G\left[\left(F \cap E_{1}\right) \times \cdots \times\left(F \cap E_{n}\right)\right] .
$$

The latter group is obviously isomorphic to some $D \sim G$ (the regular wreath product), where $D \leqslant E$. From $G(P(1) \times \cdots \times P(m))=F_{0} \leqslant F$ it is now easily inferred that $D$ must contain a Sylow $p$-subgroup $Q$ of $E$. In particular, $p$ divides the order of $D^{*}$, the base group of $D \sim G \cong F$, and from this it is readily deduced that $G \cong F /\left(F \cap E^{*}\right) \cong(D \sim G) / D^{*} \in \mathscr{F}(p)$, which contradicts our choice of $G$.

We have not been able to answer the following

QUESTION. Which saturated formations $\mathscr{F}$ (with $\mathscr{F}(p)=\mathscr{F}$ for any $p \in \chi(\mathscr{F})$ ) do actually enjoy the property that $\langle F \cap S, T\rangle=\langle F \cap S, F \cap T\rangle$ whenever $G \in$ $\mathscr{E}, F \in \operatorname{Proj}_{\mathscr{F}}(G)$, and $S, T \unlhd \unlhd G$ ?

\section{References}

[1] M. Aschbacher and L. Scott, 'Maximal subgroups of finite groups', J. Algebra 92 (1985), 44-80.

[2] R. Baer and P. Förster, Einbettungsrelationen und Formationen endlicher Gruppen, in prepara tion.

[3] P. Förster, 'Projektive Klassen endlicher Gruppen I. Schunck- und Gaschützklassen', Math. Z. 186 (1984), 149-178.

[4] P. Förster, 'Projektive Klassen endlicher Gruppen IIb. Gesättigte Formationen: Projektoren', Arch. Math. 44 (1985), 193-209.

[5] P. Förster, 'A note on primitive groups with small maximal subgroups', Publ. Sec. Mat. Univ. A utònoma Barcelona, 28 (1984), 19-27.

[6] W. Gaschütz, 'Über modulare Darstellungen endlicher Gruppen, die von freien Gruppen induziert werden', Math. Z. 60 (1954), 274-286.

[7] W. Gaschütz, 'Existenz und Konjugiertsein von Untergruppen, die in endlichen auflösbaren Gruppen durch gewisse Indexschranken definiert sind', J. Algebra 53 (1978), 389-295.

[8] B. Huppert, 'Zur Theorie der Formationen', Arch. Math. 19 (1968), 561-574.

[9] L. G. Kovács, 'Twisted wreath products and primitive groups', in preparation. 
[10] J. Lafuente, 'Eine Note über nichtabelsche Hauptfaktoren und maximale Untergruppen einer endlichen Gruppe', Comm. Algebra 13 (1985), 2025-2036.

[11] J. Lafuente, 'On restricted direct wreath products of groups', Arch. Math. 43 (1984), 208-209.

[12] K.-U. Schaller, Über Praegruppen in endlichen auflösbaren Gruppen (Habilitationsschrift, Kiel 1978).

[13] Ti Yen, unpublished.

[14] H. Wielandt, 'Sylowgruppen und Kompositionsstruktur', Abh. Math. Sem. Univ. Hamburg 22 (1958), 215-228.

\section{Department of Mathematics I.A.S.}

The Australian National University

GPO Box 4

Canberra, A. C. T. 2601

Australia 